Vol.11, No.3(2020)

Research Paper

20204477

\title{
Lateral Localization via LIDAR-Based Road Boundary Extraction on Community Roads
}

\author{
Wataru Furuse ${ }^{1)}$ Takuma Ito ${ }^{1)}$ Kyoichi Tohriyama ${ }^{2)}$ Minoru Kamata ${ }^{1)}$ \\ 1) The University of Tokyo \\ 5-1-5, Kashiwanoha, Kashiwa, Chiba, 277-8589, Japan \\ 2) Formerly, Toyota Mortor Corporation. \\ 1-4-18, Koraku, Bunkyo, Tokyo, 112-8701, Japan
}

Received on June 26, 2020

\begin{abstract}
To realize an automated driving system which is applicable to community roads, we proposed a lateral localization method focusing on road boundaries. The proposed method extracts road boundary candidates from LIDAR data by focusing on features of each road boundary, and accumulates them in the temporary memory. After traveling a certain distance, the proposed method determines road boundaries on both sides of the road by using the accumulated road boundary candidates; further, the system conducts localization. To evaluate the proposed method, we conducted experiments on community roads, and confirmed that the proposed system correctly estimated self-location.
\end{abstract}

KEY WORDS: Safety, Intelligent vehicle, Road environment recognition, LIDAR, Localization, Community road (C1)

\section{Introduction}

In recent years, the use of automated driving technologies has been attracting attention as solutions to various social problems related to automobiles. Practical applications of automated driving technologies require map information, and most of the current mainstream automated driving methods use high-definition 3Dmap. However, because preparations of such digital maps require a great deal of time and cost, we cannot apply such methods for practical applications throughout the country. To solve the problem, LeanMAP was proposed as a new map in the previous study $^{(1)}$. Because LeanMAP is based on the road network of existing car-navigation maps, it can be prepared via relatively small resources ${ }^{(2)}$. In addition, the characteristic point of LeanMAP is that it assumes a relatively simple sensor configuration.

To use digital maps for automated driving, technologies for estimating the position of a vehicle on digital maps, which are called localization, are needed. In the LeanMAP, the position is expressed as a combination of longitudinal and lateral positions along the path, and they are estimated independently. The previous study ${ }^{(1)}$ proposed a method to update the lateral position by detecting lane markers, and confirmed the feasibility of the proposed method on main roads where the clear lane markers exist regularly. However, update methods of lateral position have not been developed for community roads without standardized road boundaries, as shown in Fig. 1. Thus, this point remains unsolved.

The goal of this study is to realize lateral localization on community roads via LeanMAP and simple on-board sensors. For the goal, we classify the road boundaries of community roads from the viewpoint of their geometric features, and propose a method to detect all types of them. Specifically, we develop a system to estimate lateral position based on LIDAR information by focusing on transverse slope changes of road surfaces and 3D structures at road boundaries. Then, we will evaluate the performance of the developed system in actual environments.

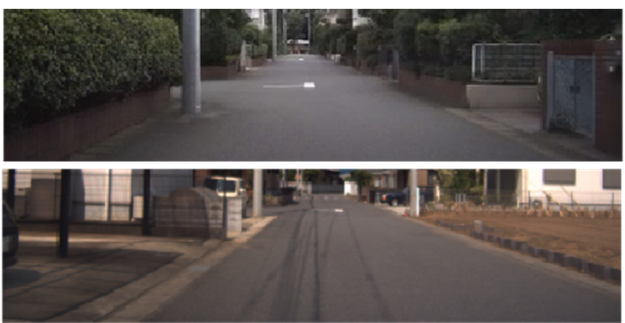

Fig. 1 Examples of community roads.

The following parts of this paper are organized as follows: the design of the system is described in chapter 2. The details of system implementation are described in chapter 3 . The system evaluation is described in chapter 4. Conclusions are described in chapter 5 .

\section{System Design}

\subsection{Lateral Localization of LeanMAP}

LeanMAP defines waypoint maps, which consist of points arranged at $5 \mathrm{~cm}$ intervals, for representing the center points along the lane. Figure 2 shows a schematic of lateral position expression of LeanMAP. The lateral position of the vehicle in LeanMAP is expressed as the combination of its lateral deviation (LD), which indicates the distance from the nearest waypoint, and its angular deviation $(\phi)$, which indicates the deviation angle between the 


\section{Wataru Furuse et al / International Journal of Automotive Engineering}

Vol.11, No.3(2020)

vehicle and the direction of the nearest waypoint. To estimate the lateral position, a previous study proposed a method to detect the left and right lane markers via an on-board camera. However, on community roads, because there are various road environments without usual lane markers, consideration of new components in the road environments as alternatives of lane markers is necessary to update the lateral position constantly. Thus, in this study, we classify the road boundaries of road environments without lane markers according to its geometric features; further, we propose a method to detect the various road boundaries instead of lane markers.

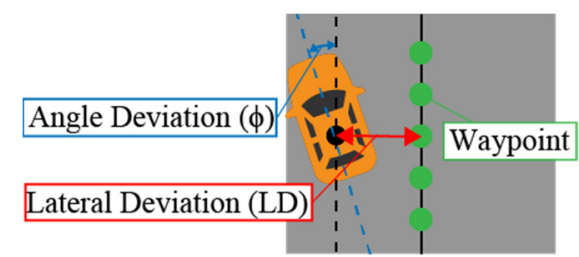

Fig. 2 Schematic of lateral position expression of LeanMAP.

\subsection{Classification of Road Boundaries by Geometric Features}

In this study, road boundaries of the community roads were classified into the following three types from the viewpoint of their geometric features.

- Road boundaries with large height difference

- Road boundaries with small height difference

- Road boundaries with varying slopes

Figure 3 shows schematics and examples of above-mentioned road boundaries. As shown in the schematic at the bottom of Fig. 3 , transverse gradient changes of road surfaces exist even at the boundary between a parking lot and the public road because the Road Structure Ordinance in Japan requires that the road has transverse slopes for drainage purposes. Therefore, we think that most roadway boundaries are classified into the above three types. In this study, we set all types of road boundaries, which include the boundaries with varying slopes, as detection targets because detecting all types of road boundaries on the community road is necessary for updating the lateral position of the vehicle regularly. However, previous studies have not proposed a method to detect road boundaries with varying slopes. Thus, we need to propose a new method to detect all types of road boundaries.

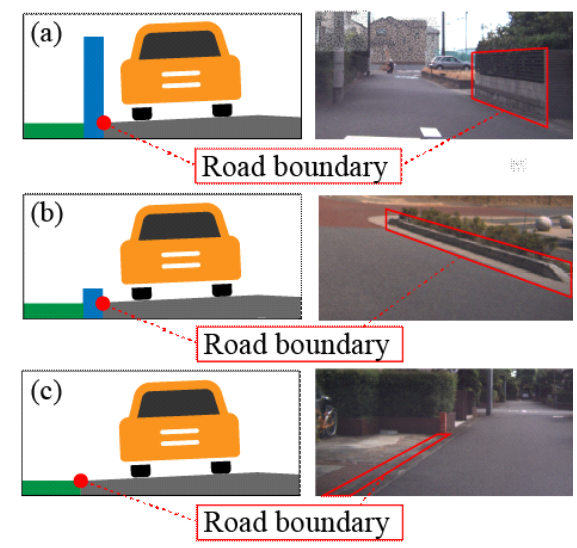

Fig. 3 Schematics and examples of classification of road boundaries (a):road boundary with large height difference (b): road boundary with small height difference (c): road boundary with varying slopes.

\subsection{System Requirements}

We considered the requirements of the system to estimate the lateral position from the perspective of achieving autonomous driving, and summarized the following three points: Required accuracy, variety of detection road boundaries, and robustness.

First, the required accuracy is determined as the following conditional expression (1) from the viewpoint that the vehicle should not deviate from the minimum width, which is $2.75 \mathrm{~m}$ on a public road in Japan. In the conditional expression, $\mathrm{LD}_{\text {error }}[\mathrm{m}]$ denotes the lateral deviation error, $\phi_{\text {error }}[\mathrm{rad}]$ denotes the angular deviation error, $\mathrm{V}[\mathrm{m} / \mathrm{s}]$ denotes the vehicle speed, $\mathrm{T}[\mathrm{s}]$ denotes the estimated period, $\mathrm{W}_{\text {road }}$ denotes the width of road, and $\mathrm{W}_{\text {car }}$ denotes the width of the vehicle. Figure 4 shows a conceptual schematic of the conditional expression. The expression considers the current lateral deviation error at a given time and the increasing lateral deviation error due to the angular deviation error by the next estimation period.

$\mathrm{LD}_{\text {error }}+\mathrm{V} \times \mathrm{T} \times \sin \left(\phi_{\text {error }}\right)<\left(\mathrm{W}_{\text {road }}-\mathrm{W}_{\text {car }}\right) / 2$

Here, we assign the minimum width of $2.75 \mathrm{~m}$ on a public road to $\mathrm{W}_{\text {road, }}$ and the width of an experimental vehicle of $1.75 \mathrm{~m}$ to $\mathrm{W}_{\text {car. }}$. As a result, the above one for the required accuracy in this study is expressed as the following conditional expression (2).

$\mathrm{LD}_{\text {error }}+\mathrm{V} \times \mathrm{T} \times \sin \left(\phi_{\text {error }}\right)<0.5$

The left side of this conditional expression will be described as the lateral self-positioning error $\mathrm{K}[\mathrm{m}]$ in the following sections.

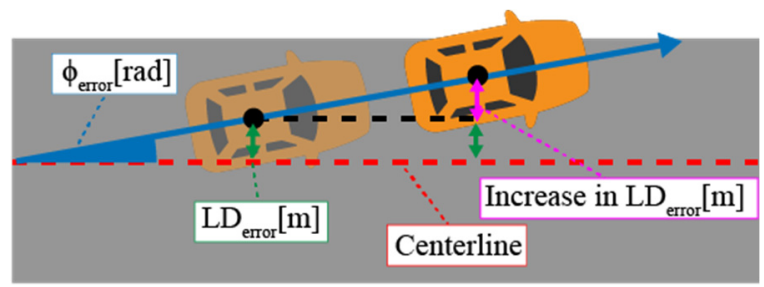

Fig. 4 Schematics of a conditional expression for a required accuracy.

Next, as for the diversity of road boundaries, we require that all road boundaries classified in section 2.2 can be detected.

Finally, the required robustness is defined as the requirement that the system can operate robustly even in the road environments with obstacles such as telegraph poles, pedestrians and parked vehicles.

\subsection{Experimental Vehicles and On-board Sensors}

Figure 5 shows an experimental vehicle and on-board sensors used in this study. Table 1 summarizes the details of the on-board sensors. The experimental vehicle is equipped with a 4-layer forward LIDAR sensor. While many previous studies on automated driving used omnidirectional multilayer LIDARs, we adopted a 4-layer forward LIDAR which is relatively simple sensor. This is because small-layer forward LIDARs are expected to be available at a lower price relatively soon. 
Vol.11, No.3(2020)

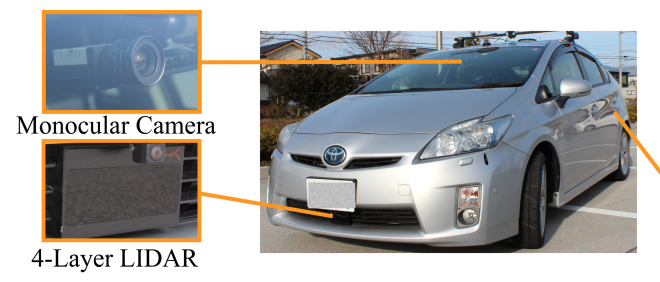

Fig. 5 Experimental vehicle.

\section{Implementation}

\subsection{Overview of the Lateral Localization System}

Figure 7 shows a schematic of the lateral localization system developed in this study. This localization system consists of the following two-step processes.

Table 1 Sensors used in lateral localization system.

\begin{tabular}{|c|c|c|c|}
\hline Sensor & Model & Maker & Purpose \\
\hline 4-layer LIDAR & LUX & IBEO & Road Feature Extraction \\
\hline $\begin{array}{c}\text { Inertial } \\
\text { Measurement Unit } \\
\text { (IMU) }\end{array}$ & $\begin{array}{c}\text { AU7428N2 } \\
100\end{array}$ & $\begin{array}{c}\text { Tamagawa } \\
\text { Seiki }\end{array}$ & $\begin{array}{c}\text { Estimation of Vehicle } \\
\text { Motion }\end{array}$ \\
\hline
\end{tabular}

\subsection{Feature Analysis of LIDAR Point Cloud}

Figure 6 shows an example of detected LIDAR point cloud. The right one in the figure shows the visualization result of the LIDAR point cloud acquired by the lower two layers of the 4layer forward LIDAR. Each point is colored according to the height of the target, and the orange dotted lines in the figure represent the left and right road boundaries and the centerline of the road. As shown in the left of Fig. 6, these LIDAR points were acquired in a road environment where a road boundary with varying slope existed at the left side and a road boundary with height difference existed at the right side. In the environment, the LIDAR point cloud has characteristics of segmental linearity in each area: the parking area (a), the left area of the road (b), the right area of the road (c), and the side area of the wall (d). In this study, we extract road boundaries and the center of the road as road feature points by utilizing the segmental linearity. However, the 4-layer forward LIDAR has a disadvantage of small amount of information per sensor cycle compared to the omnidirectional multilayer LIDAR. Therefore, we need to design a lateral localization method with taking this disadvantage into account. Specifically, in this study, we propose a method which consists of following two-step processes to solve the above-mentioned disadvantageous. In process 1 , we extract road feature points by focusing the segmental linearity of LIDAR point cloud. In process 2 , we accumulate the feature points in each cycle, and estimate the lateral position of the vehicle from the accumulated feature points.
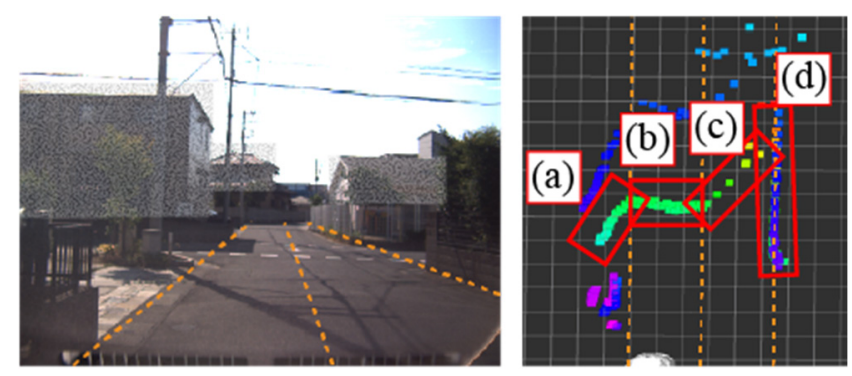

Fig. 6 An example of detected LIDAR point cloud.

- Road feature extraction process (Process 1 in Fig. 7)

The system extracts the road feature points of the road boundary parts and the road center parts among the point cloud obtained by the bottom layer laser of the LIDAR.

- Road centerline determination process (Process 2 in Fig. 7)

The system accumulates the road feature points in each cycle of the process 1 and determines the road centerline from the accumulated road feature points. The road centerline means a straight line at the same distance from the left and right boundaries of the road.

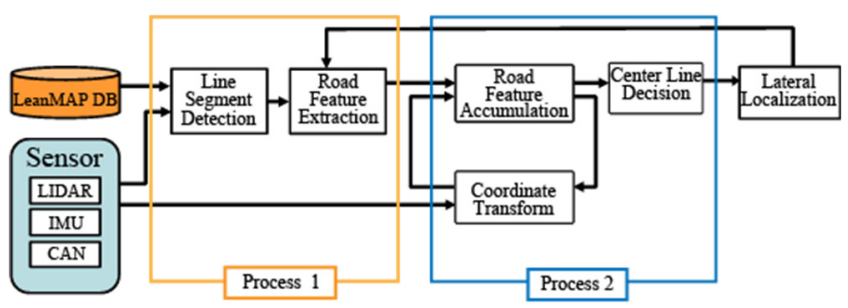

Fig. 7 Conceptual schematic of flow of lateral localization system.

Then, the lateral position is estimated from the positional relationship between the vehicle and the road centerline estimated by the processes. Although there are various road environments, the target environment of this study is limited to straight roads as a first step. In the process of lateral position update, a vehicle coordinate system is used with the center of gravity of the vehicle as the origin, the $\mathrm{x}$-axis in the direction of travel of the vehicle, and the $y$-axis in the left-hand direction of the vehicle.

\subsection{Road Feature Extraction Process}

In this process, the system extracts the feature points among the point cloud obtained by the bottom layer laser of the LIDAR. The proposed method is novel in that it can also detect the road boundaries with varying slopes, which most of the existing methods $^{(3)(4)}$ to extract lane feature cannot detect. First, the system detects line segments focusing on the segmental linearity of the acquired LIDAR point cloud. Figure 8 shows a conceptual diagram of line segment detection. The LIDAR point cloud is represented by green circles and the detected line segments are represented by red line. This line detection process is based on a parameter estimation method called RANSAC ${ }^{(5)}$, which is robust for the existence of outliers. The details of the procedure are shown below and Fig. 9 shows the schematic of a part of line segment detection. The following line detection process applies to each point cloud obtained by the two lower layers of LIDAR. 
Step 1: Determining the parameters of the candidate line, $y=a x+b$ (Step 1 in Fig. 9)

The system randomly selects two points from the point cloud of the LIDAR, and determines the parameters $a$ and $b$ of the line passing through the two points.

Step 2: Extracting neighbor points of a candidate line (Step 2 in Fig. 9)

The system extracts all points satisfying the following expression as neighbor points. Note that the coordinates of each point are expressed as $\left(x^{\prime}, y^{\prime}\right)$.

$$
\frac{\left|a x^{\prime}-y^{\prime}+b\right|}{\sqrt{a^{2}+1}}<0.8
$$

Step 3: Clustering of neighbor points (Step 3-1, 3-2 in Fig. 9)

If the Euclidean distance between two points belonging to different clusters is less than or equal to $1.0 \mathrm{~m}$, the system merges the clusters to which these points belong (Step 3-1 in Fig. 9). After that, a candidate line segments are determined from the cluster with the largest number of points (Step 3-2 in Fig. 9). The endpoints of the line segment are determined from the highest and lowest index points in the cluster. The system orders the number of indexes counterclockwise as seen from the vehicle.

Step 4: Determination of the line segment with the largest number of neighbor points

The system repeats the processes of form Step 1 to Step 3 a certain number of times and determines the line segment with the largest number of neighbor points among the candidate line segments. We call this line segment a maximum line segment. This step extracts one of red lines in Fig. 8. Then, the system deletes the points that make up the maximum line segment.

Step 5: Extraction of the detected line

The system repeats the processes from Step 1 to Step 4 . The end condition is that the number of points constituting the maximum point segment is 5 or less. This step extracts all red lines in Fig. 8.

Because the above-mentioned line detection process is based on RANSAC, the process has the advantage that it can solve the large variation of LIDAR point cloud. In addition, the system can work robustly even when the continuity of the point cloud is lost due to obstacles such as telegraph poles and pedestrians.

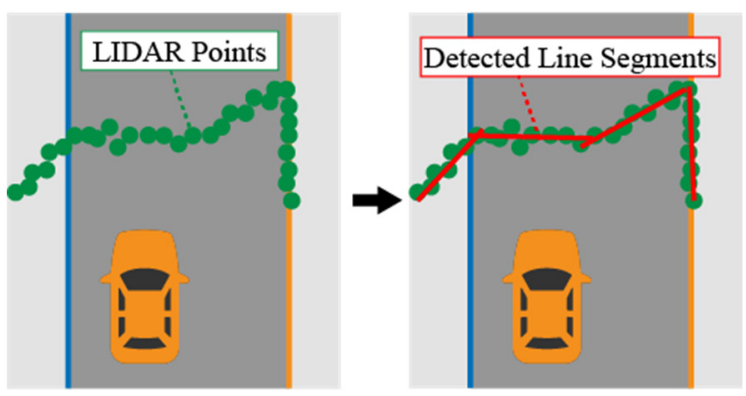

Fig. 8 Conceptual schematic of line segment detection.
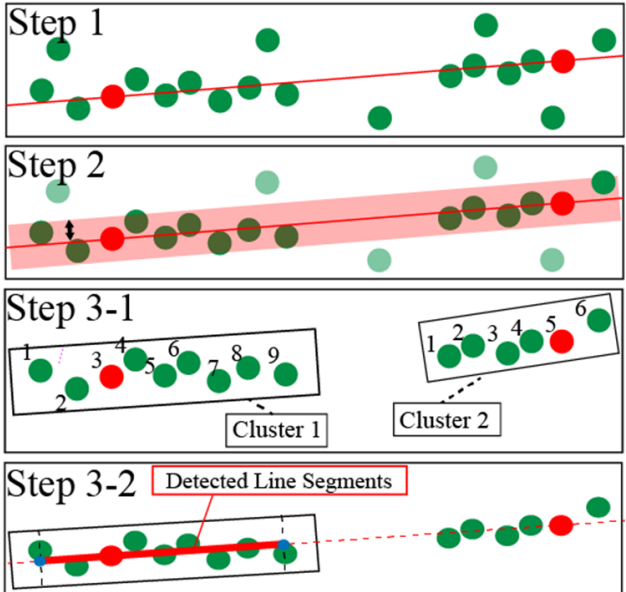

Fig. 9 Conceptual schematic of decision of candidate line segments.

Next, road boundary parts and road center parts are determined from the geometric relationship among the detected line segments and the positional relationship between the line segments and the vehicle. Figure 10 shows the conceptual schematic of this process.

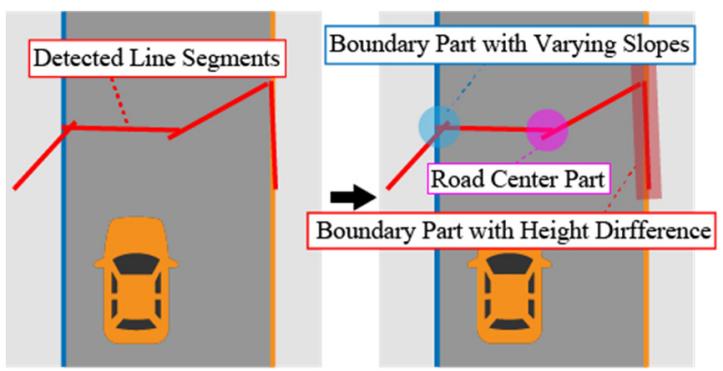

Fig. 10 Conceptual schematic of road feature part decision.

First, the system considers line segments which satisfy the following conditions as the boundary parts with height difference (red area in Fig. 10).

- The angular difference from the road centerline determined in the previous estimation cycle is within the threshold of 10 degrees.

- Line segments which are judge to be the same line segments to a certain line segment detected in point cloud of the second lowest layer of LIDAR.

Note that two line segments $\left(y=a_{1} x+b_{1}, y=a_{2} x+b_{2}\right)$ which satisfy the following two conditions are considered to be the same line segments.

$\left|\frac{a_{1}-a_{2}}{1+a_{1} a_{2}}\right|<\tan \left(2^{\circ}\right)$

$\left|\frac{\left|b_{1}\right|}{\sqrt{a_{1}^{2}+1}}-\frac{\left|b_{2}\right|}{\sqrt{a_{2}^{2}+1}}\right|<0.1$

Then, for line segments other than above ones, the system calculates intersection points between adjacent lines, and extracts these points as the transverse gradient change points. Among the transverse gradient change points, those with convex shapes from the direction of the vehicle are determined as road boundary parts with varying slope (light blue area), and those with concave shapes are determined as road center parts (pink area). 
Vol.11, No.3(2020)

Finally, the system converts road boundary parts and road center parts obtained by the above-mentioned process into road feature points which are suitable for accumulating observation results and determining the road centerline. The conceptual schematic of this process is shown in Fig. 11. First, the road is divided into left and right areas with the road centerline estimated in a previous cycle. Next, we extract the calculated transverse gradient change points around the center of the road as road center points (pink points in Fig. 11). Then, the system classifies road boundary parts into left boundary parts and right ones and converts them into left road feature points (yellow points in Fig. 11) and right road feature points (blue points in Fig. 11), respectively. In this case, a road boundary part with height difference are transformed into points at regular intervals on the corresponding line segments. A road boundary part with varying slopes are transformed into 4 points near the transverse gradient change point, considering the balance of the number of points accumulated in each road boundary part. These extracted road feature points possess $2 \mathrm{D}$ position information $(\mathrm{x}, \mathrm{y})$ in the vehicle coordinate system.

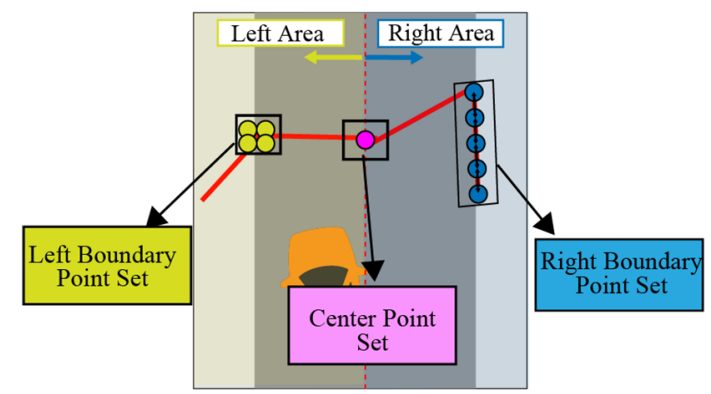

Fig. 11 Conceptual schematic of road feature points extraction.

\subsection{Road Centerline Determination Process}

In this process, the system accumulates road feature points which are obtained in each road feature extraction process, and determines the road centerline from them. The accumulation process of road feature points consists of updating the accumulated road feature points and adding newly extracted road feature points. The road feature points are updated by the equation (6) based on the vehicle movement $(\Delta x, \Delta y, \Delta \theta)$ calculated by the on-board speed sensors and IMU sensors. Note that the coordinates of the recognition result before the update are $(\mathrm{x}, \mathrm{y})$ while those after the update are $\left(\mathrm{x}^{\prime}, \mathrm{y}^{\prime}\right)$.

$$
\left(\begin{array}{l}
x^{\prime} \\
y^{\prime}
\end{array}\right)=\left(\begin{array}{cc}
\cos (-\Delta \theta) & -\sin (-\Delta \theta) \\
\sin (-\Delta \theta) & \cos (-\Delta \theta)
\end{array}\right)\left(\begin{array}{l}
x-\Delta x \\
y-\Delta y
\end{array}\right)
$$

However, such accumulated road feature points based on the amount of vehicle movement tend to accumulate errors simultaneously as the travel distance increases. Therefore, after the coordinate transformation, we delete the points located a certain distance behind the vehicle. At the same time, we reduce the weights $\mathrm{w}_{\mathrm{i}}$ of each road feature point at a constant reduction rate for each coordinate transformation. In other words, the system decreases the weight of the older points.

Then, we determine the road centerline from the accumulated road feature points. The conceptual schematic of this process is shown in Fig. 12, where the yellow, blue, and pink points are the accumulated left, right, and center road features, respectively. The procedure of determining the centerline from these road feature points is summarized below.

I. Random generation of candidate road boundary line

The system selects two points from either the left or right road feature point set, and defines a straight line passing through the two points as the candidate road boundary line.

II. Construction of candidate line combination

The system constructs opposite candidate road boundary line and candidate road centerline based on the road width information from leanMAP database.

III. Calculation of lane evaluation value

The system calculates the lane evaluation value $E$ for the combination of three lines obtained in above processes. The lane evaluation value $\mathrm{E}$ is calculated by the evaluation function that considers the number of neighbor points, their distances from candidate lines, and their weight. Specifically, the lane evaluation value $\mathrm{E}$ is calculated by the following equations (7) and (8). Note that $\mathrm{w}_{\mathrm{i}}$ in equations (7) and (8) denotes weight of a point considering the accumulation error due to the coordinate transformation, and $d_{i}$ denotes the distance between the candidate line and the point. $\mathrm{W}$ denotes a constant value determined by the width of variation of extracted road feature points. For example, the larger the variability of the points is, the larger the $\mathrm{W}$ is.

$$
\begin{aligned}
& E=\sum_{i}^{\text {All Points }} f\left(w_{i}, d_{i}\right) \\
& f\left(w_{i}, d_{i}\right)=\left\{\begin{array}{cc}
0 & \left(d_{i}>W\right) \\
w_{i}\left(1-\left(\frac{d_{i}}{W}\right)^{2}\right)^{2} & \left(d_{i}<W\right)
\end{array}\right.
\end{aligned}
$$

By repeating the above processes for a certain number of times, we determine the combination of three lines with the highest lane evaluation value $\mathrm{E}$. When the road evaluation value $\mathrm{E}$ is higher than the threshold value, we determine the candidate road centerline in the combination as the estimated road centerline.

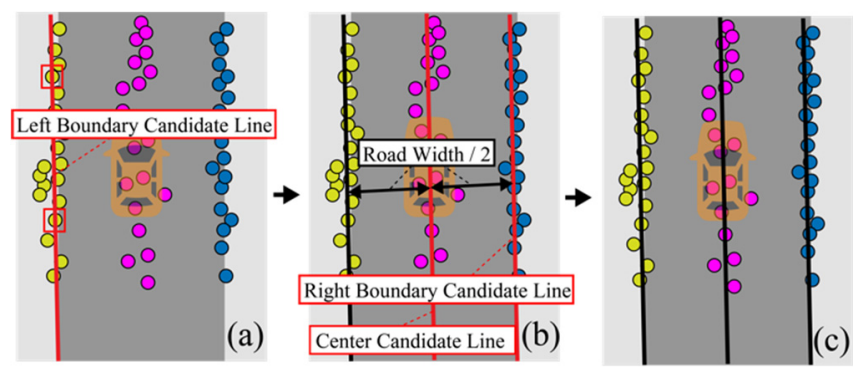

Fig. 12 Conceptual schematic of centerline decision.

Because this process evaluates the combination of the both road boundary lines and the road center line, it is possible to determine the road centerline from the well-accumulated road feature point set, even if some of the road feature points are partially lacked due to obstacles. In addition, the lane evaluation value $\mathrm{E}$ solve the problem that the variation of the accumulated points is large and accumulation errors increase according to passing time. 


\subsection{Estimation of Lateral Position}

In this process, we estimate the lateral position of the vehicle from the road centerline estimated in Section 3.3. Figure 13 shows a conceptual schematic of this process. The lateral deviation (LD) and angular deviation $(\phi)$, which are the lateral position expression of LeanMAP, are calculated by the following equations. Not that $\mathrm{y}=\mathrm{ax}+\mathrm{b}$ is the estimated road centerline.

$L D=\frac{-b}{\sqrt{a^{2}+1}}$

$\phi=-\arctan (a)$

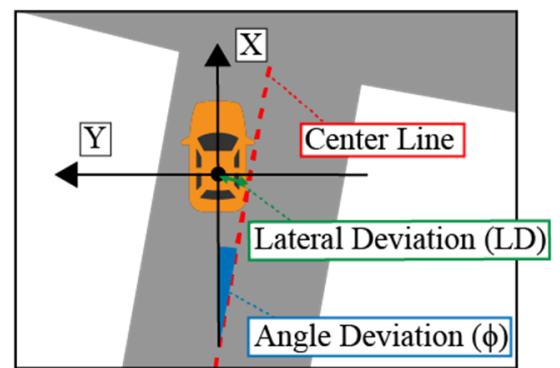

Fig. 13 Conceptual schematic of lateral position estimation.

\section{Performance Evaluation Experiment}

As for the following experiment, we asked a member of the institutional review board (IRB) for human studies of the University of Tokyo about the necessity of the review by IRB regarding the experimental plan because we recorded the image data on the public roads. However, the member of the IRB answered that we did not need to be reviewed from a viewpoint of human studies because this experiment aimed to develop the system and not to evaluate the participants. Thus, we only took care of the privacy of the people who were visible in the recorded data from the viewpoint of the personal information protection. In addition, the picture used in this paper has been processed in the residential area to protect privacy.

\subsection{Overview of the Experiment}

To evaluate the performance of the developed localization system under actual environments, we conducted an off-line experiment to estimate the lateral position of vehicle by collecting driving data on real community roads. We set up two experimental courses. Course 1 mainly consists of road boundaries with large and small differences that are considered relatively easy to detect. Course 2 includes many road boundaries with varying slope that are considered relatively difficult to detect. Figures 14 and 15 show the experimental course maps and photographs of the road environment of courses 1 and 2, respectively. In addition, examples of obstacles included in the collected driving data are shown in Fig. 16. In this way, we selected road environments with obstacles such as telegraph poles, oncoming vehicles, parked vehicles, pedestrians, and bicycles.
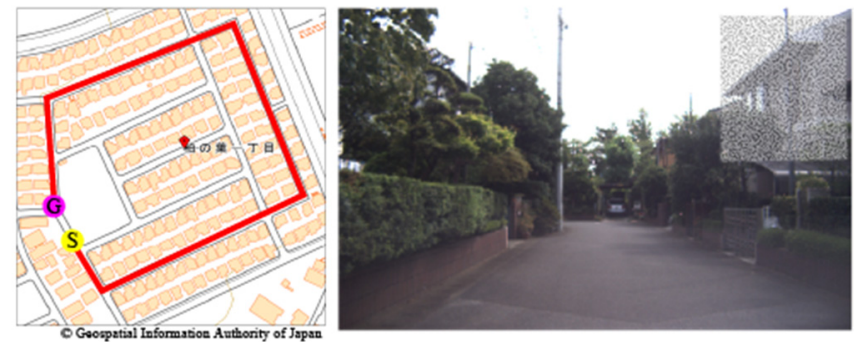

Fig. 14 Map and example of course 1. ${ }^{(6)}$
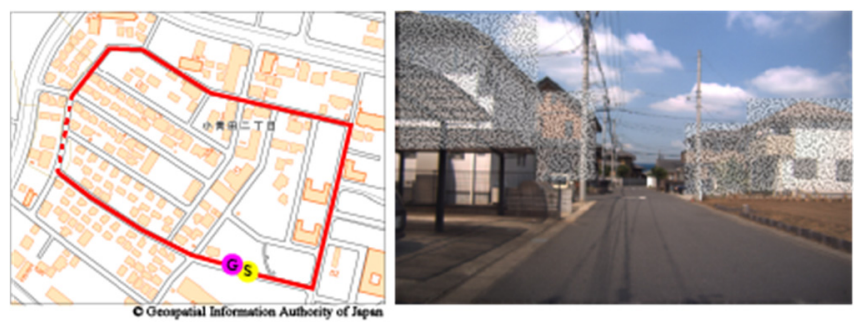

Fig. 15 Map and example of course 2. ${ }^{(6)}$
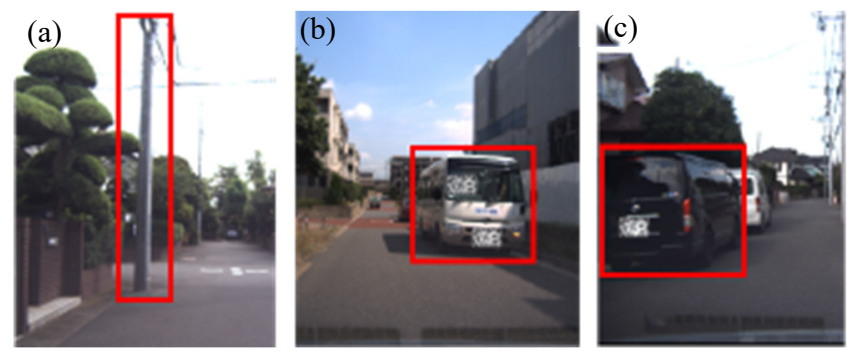

Fig. 16 Schematics of road boundaries

(a):telegraph pole (b): oncoming vehicle (c): parking vehicle.

The system estimated the lateral deviation and angular deviation from the data of 20 trips and 15 trips in courses 1 and 2, respectively. An example of the lateral position estimation in this experiment is shown in Fig. 17, where the left image is in-vehicle camera image and the right image is the visualization result of the accumulated road feature points, estimated road boundary lines, and road centerline, respectively. The color of each road feature points in this figure represents the weight of the point considering the coordinate transformation.
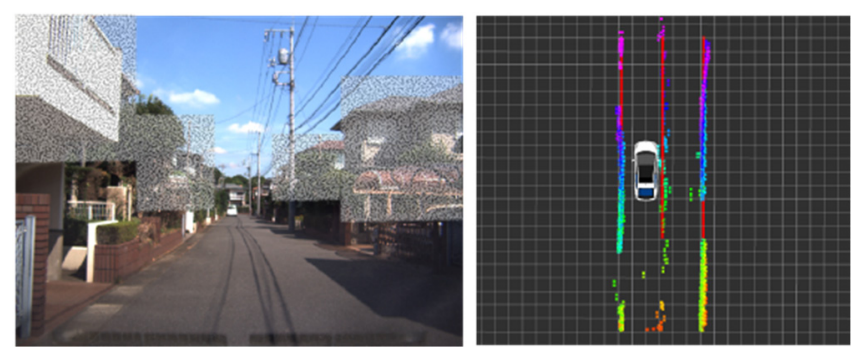

Fig. 17 An Example of processing of lateral localization system.

\subsection{Performance Evaluation of Lateral Position Estimation System}

The performance of the developed system was evaluated from the viewpoint of the following three system requirements, which are summarized in Section 2.3: Required accuracy, diversity of detected road boundaries, and robustness. 
Vol.11, No.3(2020)

\section{Required accuracy}

We evaluated the accuracy of the system by taking the lateral position acquired by RTK-GNSS (real-time kinematic GNSS) on the vehicle as the true value. Figures 18 and 19 show the histograms of the lateral position error $\mathrm{K}$, which are discussed in the section 2.3, in courses 1 and 2. Regarding the variables for calculating $\mathrm{K}$, the vehicle speed $\mathrm{V}[\mathrm{m} / \mathrm{s}]$ was assigned $8.33[\mathrm{~m} / \mathrm{s}]$ from the principle speed limit on the community roads. Similarly, we assigned $0.1[\mathrm{~s}]$ to $\mathrm{T}[\mathrm{s}$ ] from the sufficiently long calculation time of the system because $\mathrm{T}[\mathrm{s}]$ varies with the number of accumulation points. We can confirm that the required accuracy of autonomous driving is met in approximately $99 \%$ of both courses.

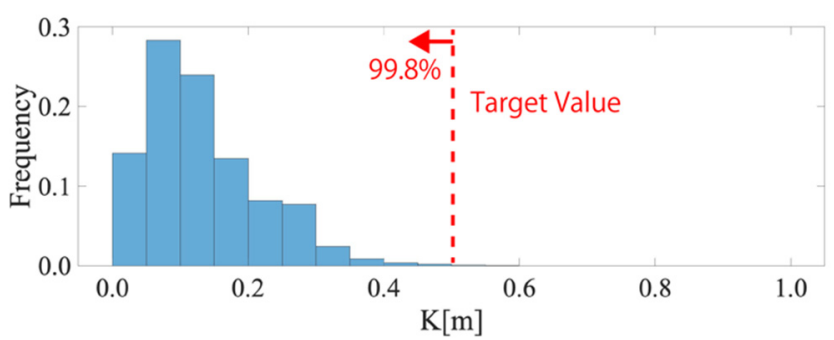

Fig. 18 Histogram of estimated lateral position error K in course 1.

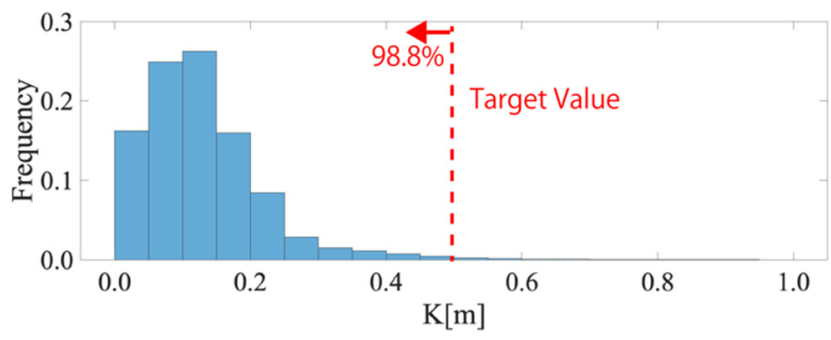

Fig. 19 Histogram of estimated lateral position error K in course 2.

Diversity of detection road boundaries

We confirmed that the proposed system had a certain accuracy in both course 1 , which mainly consists of road boundaries with large and small height difference, and course 2, which includes many road boundaries with varying slopes. Thus, we thought that the proposed system is a system that can detect all types of road boundaries classified by geometric feature. Figures 20 and 21 show the visualization results of the line segments detected from the point cloud of LIDAR in course 1 and course 2, respectively. The left images show the images of the in-vehicle camera. In the right images, white points indicate the point cloud of the bottom layer of the LIDAR and red lines indicate line segments detected by the proposed system. These figures indicate that line segment detection system works properly in both road environments. Because our proposed system extracts road feature points from the geometric relationship among the detected line segments and the positional relationship between the lines and the vehicle, we supposed that the system can extract road feature points appropriately. This line detection system is effective except for the situations where the point cloud of LIDAR on the road surface cannot be obtained due to the influence of the pitch angle variation of the vehicle.
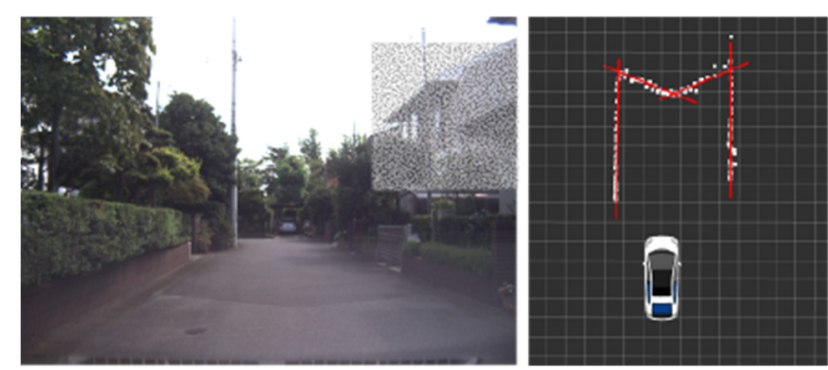

Fig. 20 An example of detection of line segments in course 1.
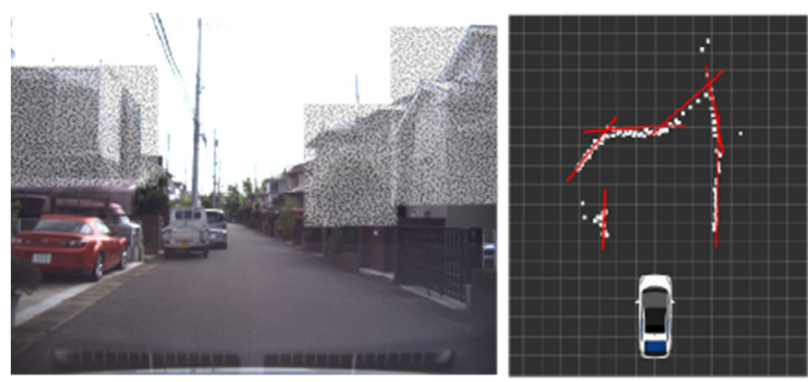

Fig. 21 An example of detection of line segments in course 2 .

\section{Robustness}

Figure 22 shows an example of the operation of the system under the condition of two parked vehicles. As shown in the figure, some of the road feature points in the region are lost due to obstacles and the system accumulates false road feature points. However, the system can estimate the road centerline and both road boundaries from the well accumulated set of road feature points. In this way, the system works robustly owing to accumulating road feature points and evaluating the combination of the both road boundary lines and the road center line.
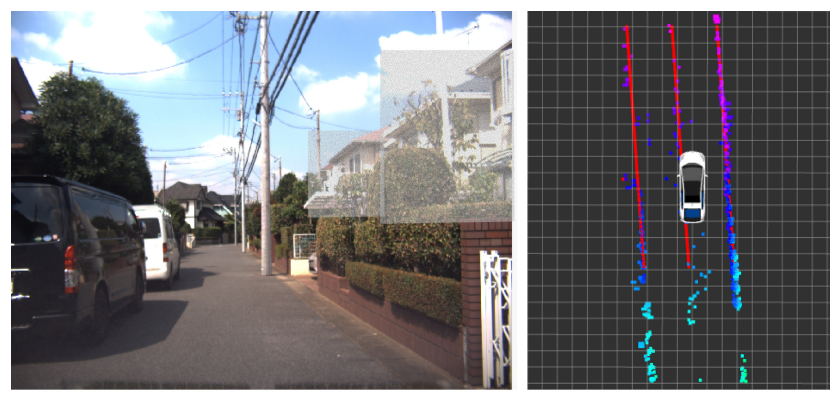

$\downarrow$ After traveling a certain distance
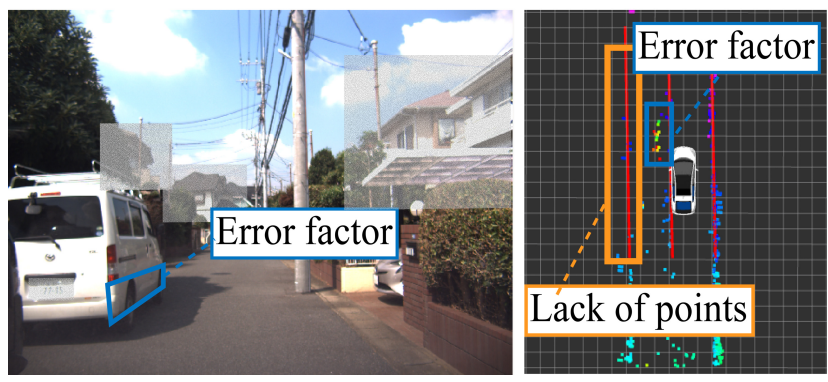

Fig. 22 An example of processing of lateral localization system in environment with obstacles. 
Vol.11, No.3(2020)

\subsection{Consideration of Error Factors in the Lateral Localization} System

Figure 23 shows a graph of estimation error $\mathrm{K}$ in the front part of the course 2 . In the graph, the vertical axis is $\mathrm{K}$ while the horizontal axis is the travel distance in the path direction. Note that we created the graph by superimposing the data of 15 trips in courses 2 . This graph suggests that the error increases mainly at the entrance of the linear section, at the exit of the linear section, and in front of intersection. We analyze the cause of this problem. Figure 24 shows the graph with the lane evaluation value $\mathrm{E}$ as the vertical axis. This graph means that the lane evaluation value $\mathrm{E}$ around the error increasing areas, which $\mathrm{K}$ become large in Fig. 23 , is small. On this point, we consider that this is due to the shortage of accumulation of road feature points. At the entrance of the linear section, there are not enough accumulation points because the system starts to extract road feature points after the curve. On the other hand, at the exit of the linear section and at the intersection, the shortage of accumulation points is caused by the absence of road boundaries in front of the vehicle. However, the problem of insufficient number of accumulated points is expected to be solved by increasing the number of LIDAR layers for extraction of the road feature points, which are currently used only in the lowest layer.

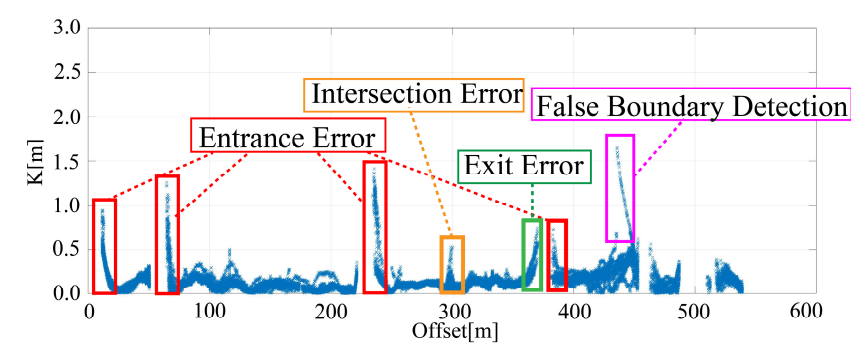

Fig. 23 Estimation error K calculated by proposed system in the front part of the course 2 .

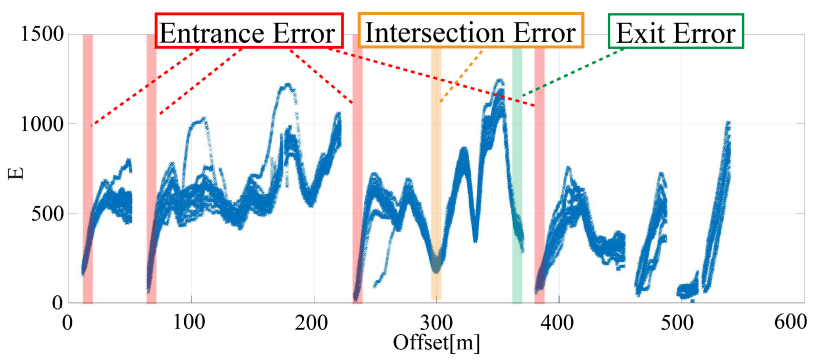

Fig. 24 Evaluation value E calculated by proposed system in the front part of the course 2 .

\section{Conclusion}

In this study, we proposed a method for estimating the lateral position in the community road by focusing on the geometric features of the road boundary parts and the road center parts. To realize this method, we have developed a system for lateral positional update by extracting and accumulating the road feature points from the point cloud of the 4-layer forward LIDAR. The feasibility of the proposed system was confirmed by performance evaluation experiments under real-world conditions.

In the future, it is necessary to extend the system to more diverse environments, including the road curve section, which was not included in this study, and to evaluate its performance.

\section{Acknowledgement}

This research has been conducted as a part of the research project "Autonomous Driving System to Enhance Safe and Secured Traffic Society for Elderly Drivers" granted by Japan Science and Technology Agency (JST), Strategic Promotion of Innovative Research and Development.

This paper is written based on a proceeding presented at JSAE 2020 Spring Congress.

\section{References}

(1) Takuma Ito et al.: Novel Map Platform based on Primitive Elements of Traffic Environment for Automated Driving Technologies, International Journal of Automotive Engineering, Vol.7, No.4, pp.143-151 (2016).

(2) Takuma Ito et al.: Data-based Modification System of LeanMAP Contents for Automated Driving, International Journal of Automotive Engineering, Vol.9, No.3, pp.115-123 (2018).

(3) Wenda Zhang: LIDAR-based road and road-edge detection, Proceedings of 2010 IEEE Intelligent Vehicles Symposium, pp. 845-848 (2010).

(4) Wentao Yao et al.: Road curb detection using 3D lidar and integral laser points for intelligent vehicles, Proceedings of 2012 the 6th International Conference on Soft Computing and Intelligent Systems, and the 13th International Symposium on Advanced Intelligence Systems, pp. 100-105 (2012).

(5) Martin A. Fischler et al.: Random sample consensus: a paradigm for model fitting with applications to image analysis and automated cartography, Communications of the ACM, pp. 381-395 (1981).

(6) GSI, "Map and Aerial Photo Browsing Service", GSI HP https://mapps.gsi.go.jp (viewed on 2020.03.05). 